\title{
QTiME - Fostering Dimensional Learning in 21st Century
}

\author{
Bala Ajjampur \\ Founder, Educationist \\ QTiME Learning \\ e-mail:www.qtimelearning.com \\ e-mail:bajjampur@gmail.com
}

\author{
Krishna V Prasad \\ SM, IEEE \\ e-mail:krishna.prasad@ieee.org
}

\begin{abstract}
Learning in 21st century is learning in knowledge era. The functions of 20th century schools and universities need to change to foster new way of learning.Atransition must be made to deal with the traits of Gen-Y and perhaps Gen-M (multigeneration) to create new ways of learning. A global reach to educational resources from anywhere and at any time, the functions of schools as information deliverers diminish significantly. To foster nonassessment based learning,there is a need to provoke learning in multiple dimensions. QTiME learning is such an attemptwith QTiME specific learningquadrantsenabled by simplistic, yet universal and actionable steps. These steps promote cyclical learning to continuously bridge the learning gaps. These QTiMEspecific dimensions, learning quadrants, the steps and their goals are a paradigm shift in learning to deliver a 21 st century learning outcomes.
\end{abstract}

Keywords - learning, education, knowledge, learning quadrants, learning dimensions, learning steps, learning outcomes

\footnotetext{
Bala Ajjampur

Founder, Educationist

QTiME Learning

e-mail:www.qtimelearning.com

e-mail:bajjampur@gmail.com
}

\section{Context}

We are in early part of 21 st century. Around the same time in 20th century, the world got realigned and got reshaped in many different ways. The industrial revolution became a definitive disruptive force to move the thinking away from the academia. It set the stage for the education of the masses to create the skilled workers of the Industrial Era. The domino effect set in motion the creation of knowledge workers of an era that we now know as 21 st century Knowledge era [20]. This knowledge era, where the knowledge is shared freely, with anyone, anywhere and abundantly, is posing a different set of challenges for the education of the masses. The big bang of the internet created a networked society of the $21 \mathrm{st}$ century that now spans the entire globe, and, is yet another disruptive force that transformed the educational landscape of the 20th century.

The skills acquired by theskilled worker got automated and were off-loaded to machines; machines became complex, intelligent and sophisticated; and, the skills to manage them became broader in scope and refined in its application. The organizations changed to address the service and the use of the products; to manage product life cycles; and to manageincreasingly efficient manufacturing processes. They sparked the creation of a whole new industry that we now know as service industry ushering in a need for new types of learning. The global connectivity, the growth of the service industry and the free sharing of knowledge across diverse communities needed a different approach. 
Itisbelieved that the community colleges of USA, a 20th century phenomenon, were firmly set in the landscape of American Education as 21 st century approached [19]. While these colleges are equipped to continue providing excellent vocational skills, still considered asessential skills for the new millennium, these skills may not be enough to deal with the 21 st century disruptive forces.

These disruptive forces started to create cracks in the foundations of 20th century learning. While it is prudent to acknowledge that a stable grounding in education was provided by the 20th century methods of delivering education, the pace of expansion and the growth of higher education needed a different approach. The efforts and methods of 20thcenturywere now in need of retooling. The needs of the knowledge workers of 21 st century became vastly differentfrom the needs of the 20 th century learners.

The speed of learning needed to increase and the capacity of delivering the education needed to increase. Engaging the learner in a world where distractions are on rise needed to be addressed. Rewarding the work performed as a service or as a product needed to be balanced.

Initiating unlearning and creating trust, in a landscape that is broadening and thickening, now needs a fresh look. 21st century learning is at its crossroads and there exists a perceived need to move the 20th century learning into other dimensions of learning that were hitherto might have been either overlooked or assumed to exist inherent in the education provided.

The paper addresses the needs for a framework that is robust, scalable and with a mass appeal for a quicker adaptation to help manage and align the disruptive forces in the direction of progress through a growth that is systemic to overcome or address a chaotic growth. Gen-Y should be able to inherit a world that continues to be responsive to the constant changes introduced by the transformational forces of global economy, connectivity and knowledge sharing [7]. Many imperatives around the world, including the SCAN's (Secretary's Commission on Achieving Necessary Skills) list from USA, formation of NSDC (National Skill Development Corporation) in India bring the necessary urgency to reinvent learning for the 21st century. QTiME learning framework is presented here as one such framework to bring in a new perspective of learning in the 21 st century by integrating learning acrossseveral learning dimensions and, claiming them as the essential 21 st century learning dimensions.

\section{Study}

To understand and acknowledge the drawbacks of traditional learning methods of 20th century, a study was made to recognize, understand and qualifythe disruptive forces at play in the 21 st century learning landscape.

The first disruptive force can be recognized as a force playing on the empty minds of today's learners. The mantra of time-saved is time-invested has now become time-saved as time-wasted scenario. These minds are now falling as an easy prey to both the distractive and the destructive forces. Trying to shape today's learning minds to an educational structure that may notaptly equip and engage them may be one such reason. Some of these destructive forces have taken the forms of bullying, school violence and in extreme cases, leading to killings and suicides (Figure 1).

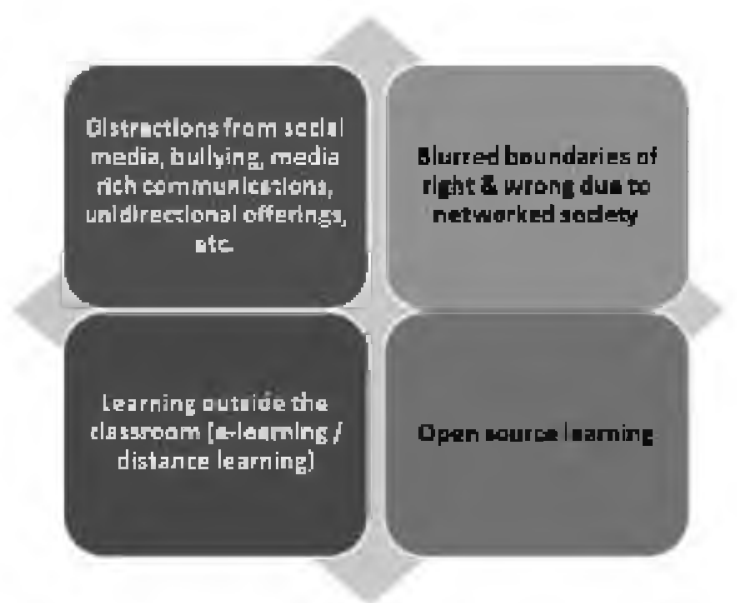

Figure 1 : Disruptive forces for traditional learning

A second disruptive force that comes to play blurs the boundaries of right and wrong and may be attributed as a by-product of the networked societies that we see today.

A third disruptive force, in the form of distant leaning or e-learning [17], is constructive in its nature, but is creating cracks in the traditional forms of learning by introducing the possibilitiesbeyond the four walls of traditional classrooms.

A fourth disruptive force, open-source learning, though leads to questionits authoritativeness, offers 
broader and rounded perception; enables the access to the content outside the curriculum; and, creates the ease of access to the masses. These changes are hurtling learners towards the possibilities of collective and collaborative learning in 21 st century[16].

The traditional form of learning falls short to fight, in a collective fashion, all such disruptive forces increasing in magnitude, as they have made the educational landscape broader, foggier and denser. The need to understand the world driven by innovations and fragmented trust cries out loudto extend the learning, mostly from outside, beyond the 20 th century reforms. The learning needs to progress into making learning to happen in dimensions that drive the learning happening in other dimensions and formulate necessary strategies to foster learning independent of the disruptive forces.

\section{Move to Multi-dimensional Learning}

We live in a three Dimensional world. We constantly absorb the three dimensional landscape that we see through our preceptor senses. We learn to deal with its changes and have learnt that this change is constant. We have come to an understanding that our learning is multi-sensory, implicit, and natural, varied but is happening and we adjust. When wethink fast to adjust, we think qualitatively and we build on that to think intuitively. However, as soon as we are asked to reason outour thinking we feel that weneedto think slow and start thinking quantitatively to support or verify that intuition. That part of the brain, now, kicks in to provideor aid us with satisfactory answers to defend our actions through rational means [12]. We forget sometimes that we are bestowed with this unique strength that directly relates to our ability to think both intuitively and rationally.

This thinking and learning varies depending on the level of Trust that we feel we have; the Innovation mindset that we think we can summon; the Methods that we are able to design to explain the actions taken; and, recognize and reward the Effort for an assessment with a quantitative rigor. The agility in learning can, then, be deemed to come from the ability to learn or make learning happen in these multiple dimensions; from the ability to understand the complex intra-relationships;and, from acknowledging the inter-relationships that inherently exist across these dimensions.

\section{Qtime Learning Premise}

QTiME learning is setting the stage for the learning to happen in multiple dimensions; for a transformative learning to begin;to shy away from proposing a reformative learning; and, to prepare the learners for the challenges of 21 st century. The drivers of the 20th century learning mayneed to take the backseat to takeadvantage of the new technologies and the new ways of communicating in

21stcentury.Websites, Wikis, Podcasts, Blogs, networking sites have already become part of this new ways of learning.

QTiME learning premise is that: New ways of learning creates new life and new life creates new learning bounded only by the limitations of the imagination.

Sir Albert Einstein's Theory of Relativity transformed our understanding of the universe in many ways [6]. Our understanding of what education and learning means has been constantly fueled by such trans formative forces even today. If it is not related to or not aligned to the world we live in today, begins to lose its significance and fuel the disruption, destruction than construction. The journey through a widening and a denser landscape is not straight forward, and, attempts to reform at the frills may not help the ecosystem to sustain itself through the changes.

QTiME learning embraces, among many things, learning quadrants as the basis to a learning conceptof dimensional learningto move away from the linearity in learning (Figure 2). QTiME moves away from a delivery model of education to masses to a delivery model where learning can be customized. The availability of new technologies, easier access to and a broader acceptance of internet and mobile technologies makes this possible. They act as catalysts

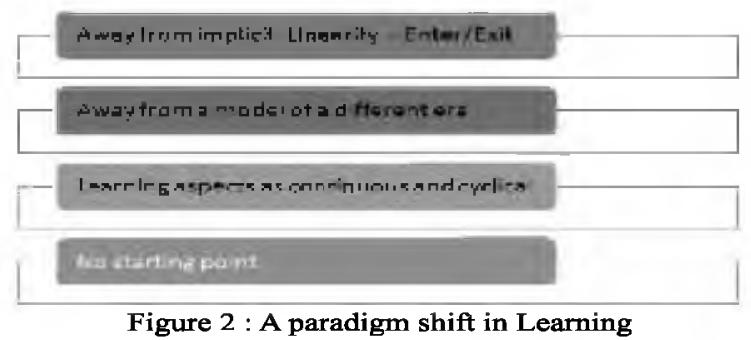

to overcome the 20th century delivery of education shortfalls. 


\section{20th Century Learning Shortfalls}

20 th century education infrastructure was built on the need to take the education to masses. The need was managed by many styles of policies adopted byboth the developing and the developed worlds. The race was to pass the knowledge and the wisdom of ages to the learners of today, and, to focus onliteracy and numeracy at the basic levels of imparting learning.

The challenges posed by the ncreasingavailability of the educational content outside the classrooms; adaptation becomingeither sketchy or in silos made the learning happening outside the classrooms to become misaligned, then, increasingly cause disconnections [2].

GERM (Global Educational Reform Movement) assessment model that was accepted globally, focused, rightly or wrongly, on a test-based learning. That might have foreshadowed the necessary learning needed in other thinking skills that could have augmented the critical thinking skills. With the advent of new technologies to embrace learning outside the classrooms, the landscape widened and expanded to reflect the connected world. These connections blossomed into much experimentation in delivering the education. But efforts remained as the extensions of the mass education to cater to more masses and might have hastened the misalignment.

No attempt was made to push the learning into other dimensions assuming learning happeningimplicitly as part of the education delivered. No attempts were made to push the learning to move it away from the model of the industrial era to the knowledge era. Instructional learning [18] was at the core of learning, when the need to reform it by an integrated learning was still being considered for implementation.

Some of the prevailing inequalities in the society can still be attributed to the low levels of learning by a section of people who do not have similar access to educationas enjoyed by those who were able to secure, yielding higher returns and continuing to createinequalities of varying proportions [21]. The sustenance ofeconomic growth through the delivery model adapted in the 20th century was becoming difficult because of such inequalities continuing to exist in the delivery of education. The inequalities remained noticeable across the sexes too, with similar gradations.
The spending on the vital service sectors like education and health remaininglow; failing to keep pace with the infrastructural needs to meet the challenges of the growing population, and, competition; failing to respond or monitor the spending that created avenues for corruption and rentseeking; failing to create the awareness in the general public that education is their right; power mongering tactics by the elite when attempts were made to decentralize the delivery of education; innovative ideas to create participation by a wider community to include parents attracting prohibitive costs; and , an expectation that the community will participate in getting the education delivered, were many of the shortfalls of 20th century education. The right to education initiatives later on, in many forms, did take the awareness to the needs of securing a good education to everyone, but the implementation was again slow because of the assumed need of having a 20 th century model of delivering education to the masses.

NGOs (Non-Government Organizations) and their presence in the educational field rivaled the success of many private organizations involved in the delivery of education. It is noteworthy to follow the operations of those NGOs who are making it possible to take the education to the remote areas of the country and others who are making it possible to incorporate the current technologies to enable the curriculum content that teachers use to deliver the education. To take education to the doorsteps of every learner, a formal enrolment does not guarantee that the education is taking place. Teacher and student absenteeism will continue to contribute to the problems faced by the 20 th century model of delivery.

\section{Qtime-A Paradigm Shift}

21 st century therefore needs a setup that explores new possibilities; flexibility to see the alternatives in the delivery of education; unlearning the old ways to embrace change; introduce new concepts in learning, to make that crucial difference to bring about the necessary transformation. One such transformation is to view schools as service centers for education. The providers need to focus on the outcomes desired and be ready to institute the needed changes in the delivery of the education and the curricula.

This can be viewed as a disruption to the education system. However, the bottom-up approach that catered to the needs of the industrial era now needs to 
change to top-down approach to cater to the knowledge era. The drivers of education of the 21 st century now reside in the thinking dimensions than in the physical dimensions. The learning has to happen in the thinking dimensions to drive the learning in the physical dimensions. The education constantly needs to be constantly aligned, by perceiving the needs of the world outside the school system to make learning inside the school system to be agile and let the changes flow all the way down to delivery at the basic levels of learning.

As culturally diverse universe unite, the differences of ethnical, racial or religious nature begin to disappear to give the clarity on the needs; frustrations and successes of an individual cannot be mirrored by other members of the group. To $\mathrm{m}$ a k e learning happen, amove away from the focus of mass education to the focus on the individual is needed to elicit peak performance that translates into derived efficiency. The attention that is divested in others is now invested in self to set the stage for the needed transformation to happen with less friction at higher than the individual levels. The global trend towards outcome assessment will affect the entire supply chain and hence it has to be addressed at all levels. Flexibility, scalability and ease of understanding to implement the changes on a continuous basis will become a necessity [19].QTiME addresses the shortcomings of the outdated curricula; the absence of continuous assessment inside andthe career advice outside;and, the problems created by the educated unemployment, by moving the delivery of education away from the model of 20 th century.

The model proposed by QTiME is a framework of continuous and cyclical learning, creating awareness by bounded and unbounded learning, introducing concepts of expanding and shrinking learning, and,of learning quadrants and learning dimensions. It makes a deliberate attempt to define the purpose and the goals of these quadrants to a simple, brief and easily recallable set of words. It employs acronyms with an effort to reachthe growing mass of learners in a widening and a denser landscape, yet giving the flexibility at higher levels of learning for interpretation. Being capable of achieving the transformations at the grass root level yet making it scalable to higher levels of learning, QTiME introduces a new paradigm in learning for the 21 st century.

QTiMEintegrateslearning on the premise that the learning is happening in different dimensions knowingly or unknowingly, intentionally or unintentionally. QTiME systemic thinking has shown that sporadic learnings can be molded into the dimensional learning. The growing number intelligent agents assisting every task that we do today; recommending the directions that we take today; give QTIME a much needed systemic thinking to be able to form the new foundation with definable inter and intra relationships. One such relationship is defined as: the delivery of learning in the driven dimensions is dependent on the learning happening inthe driving dimensionsand each dimension has a defined outcome (Figure 3).

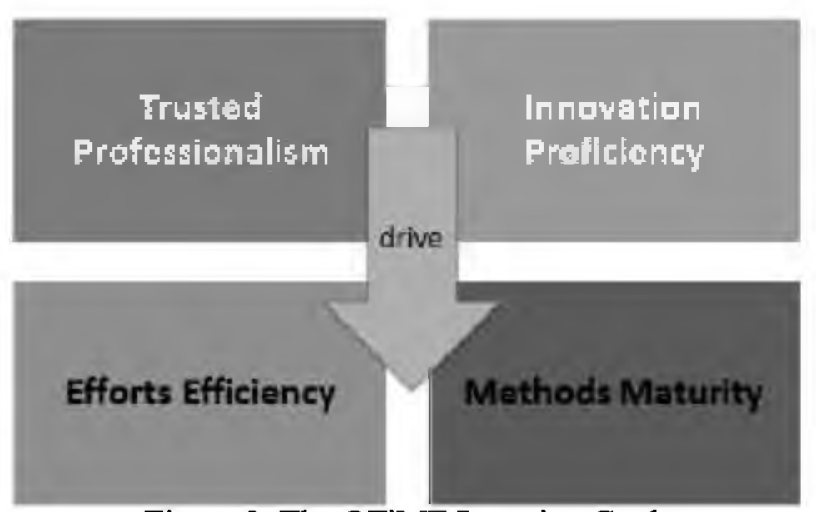

Figure 3: The QTiME Learning Goals

QTiME identifies the Trust and Innovation Dimensions as the driving dimensions belonging to the thinking dimensionsand the Method and Effort dimensions as the driven dimensions belonging to the physical dimensions. While the yardsticks to measure the effects of the interactions of these dimensions in a way QTiME is proposing is still in its infancy, a qualitative analysis can still be made to ascertain the values realized. QTiME is setting the stage for further investment in this research and is not yet ready to claim that it provides a permanent cure to all the problems or shortfalls identified earlier in this paper. But, it is ready to make the claim that it's all-inclusive and is poised to provoke learning in essential dimensions that is explicit and systemic. As we progress into 21 st century, it is possible to discover more than those claimed here as essential starting dimensions.

There is scope to argue and to make claims on the needs of defining and identifying the learning quadrants towards fulfilling the ultimate goals of each dimension. Evidence already exists now to accept these quadrants qualitatively on general terms to reserve the application of the quantitative rigor them 
at a later stage. Further research and studies over an ascertainable period of time is required and will have the quantitative proof that is necessary to broaden the QTiMEacceptance and implementation.One such necessity is analyzing achievement gaps across multiple dimensions. An achievement gap in a testbased assessment of learning has been known to depend on several other factors that influence learning and teaching [14]. There is a need to create measurement yardsticks for these influencing factors to assess learning outcomes and measureon a continuous basis[10].

QTiMEpromotes learning in multiple dimensions consciously and simultaneously to fuse the qualitative learning to the quantitative rigor and to fuse the fast thinking to slow thinking. It makes learning purposeful, and most importantly provides structure to assess the gaps to fast-track learning and toamplify innate talents. The uniqueproperties of the learning quadrants along with the proposed actionable steps provide the basis for an integrated learning that is multi-dimensional, overarching, and inclusive.QTiMEis setting the stage for a transformative learning than a reformative learning.

\section{Qtime Approach}

A. Trusted Profesinalism through Trust Quadrants: Many quantifications of trust attribute have been attempted. While it is easy to acknowledge that the element of trust almost always involve human beings, it permeates across many non-human devices above and beyond the established notion of any type of outcome. When trust involves human beings, the underlying perception that the speed with which a task can be done relates directly to the propensity to trust is irrefutable [1].

Irrespective of the percentage of human participation, the institutions and societies exist to serve the needs of humans and their trust levels play a role in learning. The two levels of trust at the individual and group levels may often be measured as intuition and instinct qualitatively, and, at the other two levels, by the established processes or the policies that are implemented. Developing a high propensity to trust at the individual and group levels, therefore, becomes a priority in delivering the education through higher level entities.

By addressing explicitly the instincts and intuitions at lower levels to bring about a good judgment to elevate the higher level processes to become trustworthy will become the primary outcome of the learning from this dimension. Trusted professionalism would be the ultimate outcome on which the learning in this dimension can be assessed as complete. Self-trust, group trust, institutional/organizational trust and the community/societal trust claim their places as learning quadrants in the trust dimension.

Quadrants of QTiME Trust Dimension are:

- Self Group

- Organizational

- Societal

B. Innovation Proficiency through Innovation Quadrants:

QTiME recognizes the learning in Innovation dimension as the game changer in driving the methods and efforts required to transform the mindset for the 21 st century learning. QTiME treats the learning in the innovation dimension as having the mindset that promotes innovative thinking. Common sense presupposes many of the established norms of perceiving innovation and is true here as well.

Robinson(2009) observes that "One of the enemies of creativity and innovation, especially in relation to our own development, is common sense". Physiologists identify four other senses that give us a sense of our being in this world as humans. While some of those can be associated to the traits of physical dimension, viewing the innovation dimension as the thinking dimension exposes other traits that are specific to making the learning happen. While new learning can wash away the old learning, in the absence of itis to make an explicit attempt to unlearn. The failure to unlearn as the world changes will be the first such errors of common sense. To create the innovative mindset, and, to reach a level of proficiency that QTiME proposes relearning, rebranding and resourcing as he other learning quadrants needed to aid the learning effort.Innovation proficiency has nothing do with innovation being small or great. It does not even presuppose that an innovation has to happen to achieve proficiency.

It is a level of proficiency required to be achieved to recognize that innovation is the result of a mindset, is a continuous process, and, is a collaborative effort. A learning mindset that causes less friction for Innovation is the first step towards achieving the proficiency. Innovation proficiency is the desired outcome of the learning in this dimension.

Quadrants of QTiME Innovation Dimension are:

- Unlearn

- Relearn

- Rebrand 


\section{- Resource}

C. Methods Maturity through Method Quadrants:

Dr. Pasi Sahlberg [5] in his talk elaborates on how theFinnish model helped Finland to ascend as one of the top five performers in the world to deliver education. He attributes that to the move away from introducing a competition through a test-based assessment environment to a collaborative environment.

Plethora of methods is available to deliver education at varying levels of maturity and they decide the outcome of many new initiatives and collaborations. A healthy competition can be viewed as collaborative in an outcome-based assessment. The outcome of collaboration is that everyone wins and the same holds good in an environment of healthy competition. Benchmarking helps to validate various methods. Applying what is learnt organizes the work.Delivery plans the work for ultimate consumption. For learning to happen or to address the gaps in learning, all method quadrants orchestrate towards the maturity ensuring the increase in the certainty of the outcome.

Quadrants of QTiME Method Dimension th e r e f o r e are:

- Apply

- Benchmark

- Collaborate

- Deliver

D. Efforts Efficiency through Effort Quadrants:

Effort itself is a hard measure as an absolute value and has undergone transformations in its measurements from as early as 18 th century from outcome based effort to time-based effort. This uniquely distinctive attribute that is not easily measurable, but perceived to be an attribute that only humans possess and are able to relate to realize transformations is at the center of learning in the effort dimension. Hard work can be elicited, but without an appropriate measure of its outcome, the effort applied will be the effort wasted. Hence, the other unique attribute of human beings called trust plays an important role in eliciting effort that is commensurate with the desired outcome. Covey (2006) observes"A significant dimension of combining high analysis with high propensity to trust is the synergy that elevates instinct and intuition to the realm of good judgment".

When motivated individuals form a group, it gets translated to the group's behavior as the motivated groupthat performs [15]. 20th century harnessed the powers of aunique group of individuals called teachers. They are the essential motivatorsto make that essential difference to impart education. A culture of providing the education that is motivated from within underpins outcome. Dr. Fox (2014) observes "Behavior is what you do (whether you're conscious of it or not). And culture is the sum of a group's behaviors. It underpins all results."

Dr. Pasi Sahlberg [5] includes culture in the productivity equation as a unique element to show that the productivity realized by effort times intelligence gets boosted or dampened by the presence of this unique cultural element in the equation.

QTiME embraces the time effort as a complimentary effortto underpin the quality of the results. The efforts even though can be made efficient, require certain amount of time to adopt efficient techniques to produce the results. This time effort encapsulates different forms including practice, seeding, vision and experimentation. Efforts efficiency is the outcome in learning from this dimension.

Quadrants of QTiME Effort Dimension therefore are:

- Provider

- Student

- Team

- Time

E. QTiME actionable Steps:

It may be perceived that for a learning to happen in many such diverse dimensions, the framework may needto be complex, varies on the desired outcome, and,there are no simplistic steps to take to carve a path through a landscape that is widening and becoming denser accelerated by the transformational changes in multitude of directions. Comparing the learning landscape to an ecosystem of a rain forest, it is understandable to expect a zigzag path through that. However, the complexity does not necessarily require complicated steps, and, may not be necessary if the natural forces are understood, if the growth is understood and if the ecosystem is understood.

QTiME neither claims nor ascertains that the actionable steps that it is proposing are the only steps needed to be taken, and are sufficient. Instead, it proposes that they are the essential first steps that must be taken in a cyclical and continuous fashion to promote and provoke learning in each of these dimensions without the need todetermine the first step. 


\section{QTiME proposed essential first steps are:}

- Acquire

- Begin

- Connect

- Discover

\section{Recap}

QTiME hasintroduced a new paradigm as a moveaway fromimparting learning on a mass level towardsthe possibilities or adjacent possibilitiesin imparting learning at individual levels; that it can develop other thinking skills that otherwise are overlooked by the over indulgent adherence in developing critical thinking skills. Critical thinking skills are essential, but may not be enough to deal with all the challenges that learning in 21 st century can present [9]. While the driving dimensions provide a basis for qualitative assessment, the maturity and efficiency of the driven dimensions createbest practicesand outcomes in the learning landscape.

The learning landscapes, even though, appear same when viewed from above, present a different scenario at the ground level. The foliage that is supporting the forest below is organic, has different growth levels, and has different types and levels of nourishment. Most importantly the overall ecosystem that is sustaining the forest will be better understood when learning progresses to learning inthe multiplicity of dimensions. QTiME learning is poised, constantly and continuously, and ready to respond to the changes in the ecosystem for growth and sustenance by creating a best possible outcome.

\section{Acknowledgements}

The initial attempt,some ten years back,was to recapture the young and talented minds, and, to find ways to move them away from distractionstowards a focused learning through the concept of Circle of Learning. The need to understand the disconnections from the world outside to the sanitized school systems of inside, increasing involvement to widen the studyfrom US School systems to include India and Australia, examining how these school systems feed and fork into two types of higher learning, academia and vocational[8] acted as catalysts to formulate QTiME concepts.

Working closely with the workings of community colleges in USAthrough MAPS program at the McHenry County College, at school level through the technological initiatives undertaken at D300 schools district, invitations received from a wide spectrum of educational entities to speak on these concepts further fueled the growth to formulate QTiME learning.

The perceived need to go beyond the critical thinking skills to explore the realms of design thinking, perceptual thinking, and constructional thinking gave birth to the current form QTiME. The methods and maturity levels achieved in Information Technology industry helped me to formulate systemic thinking to explore and understand the chaotic growth of learning platforms. QTiMEis now ready to propose this new thinking as a paradigm shift in learning to deliver education in the 21 st century.

\section{References}

[1] Covey, Stephen M.R.; Merrill, Rebecca R. (2006). The SPEED of Trust: The One Thing that Changes Everything (p. 290). Free Press. (Trust Dimension).

[2] Dewey, John (2012). Democracy and Education: an introduction to the philosophy of education (p. 10).

[3] Robinson, Ken; Lou Aronica (2009). The Element: How Finding Your Passion Changes Everything (p. 32). Penguin Books Ltd. (Innovation Dimension).

[4] Fox, Jason (2014). The Game Changer: How to Use the Science of Motivation With the Power of Game Design to Shift Behaviour, Shape Culture and Make Clever Happen, Wiley. (Effort Dimension).

[5] YouTube link on Dr. Pasi Sahlberg talk delivered at Graduate School of Education, University of Melbourne "How Finland remains immune to GERM"'.

[6] Barry R. Parker 2004, Albert Einstein's Vision: Remarkable Discoveries That Shaped Modern Science, Prometheus Books.

[7] Vibiana Bowman Cvetkovic; Robert J. Lackie 2009,Teaching Generation M, Neal-Schuman Publishers.

[8] Lynn Olson 1997, The School To Work Revolution, Da Capo Press.

[9] Edward De Bono 2008, Think - Before It's Too Late, Vermilion.

[10] Richard A. Voorhees 2001, Measuring What Matters, Jossey-Bass.

[11] A.C. Grayling 2010, Ideas That Matter, Basic Books. 
[12]Kahneman, Daniel (2011), Thinking, Fast and Slow, Random House Digital, Inc.

[13] Mike Seymour 2004, Educating For Humanity: Rethinking The Purposes Of Education, Paradigm Publishers.

[14] Karen Miller 2010, The Achievement Gap, Green haven Press.

[15] John C. Maxwell 2001,The 17 Indisputable Laws Of Teamwork: Embrace Them And Empower Your Team, Thomas Nelson.

[16] Satnam Alag 2008, Collective Intelligence In Action, Manning Publications.
[17] Kenneth Fee 2009, Delivering E-Learning: A Complete Strategy For Design, Application And Assessment, Kogan Page Ltd.

[18] Kevin B. Zook 2000, Instructional Design For Classroom Teaching And Learning, Wadsworth Publishing.

[19] Arthur M Cohen, Florence B Brawer (1994), Managing Community Colleges, Jossey-Bass.

[20] Thomas A Steart, 1997, Intellectual Captial, The new wealth of Organizations, Doubleday.

[21] Stuart Corbridge, John Harriss, Craig Jeffrey (2013), India Today, Economy, Politics and Society, Polity. 